\title{
La conexión Onetti-Saer: la arbitrariedad del narrador en Los adioses y Glosa
}

\author{
Christian Claesson**
}

\begin{abstract}
Resumen
El punto de partida de este artículo es un ensayo, publicado en 2005 , en el que Juan José Saer comenta los conocidos párrafos iniciales de Los adioses (1954), de Juan Carlos Onetti. En estos primeros párrafos de la novela se establece el contrato de lectura entre narrador y lector, pero sus fundamentos tambalean: al mismo tiempo que el narrador reivindica su autoría ( $y$ autoridad) en términos despóticos, también la declara completamente relativa. De esta forma se hace una serie de preguntas importantes, de las cuales una es crucial: ¿cuál es el papel del narrador de una historia? La misma pregunta caracteriza a Glosa (1986) de Juan José Saer, una novela que se centra en la transmisión (poco fiable) de una narración. La autoridad inestable del narrador es socavada por una serie de paratextos importantes, que resuenan a través de la novela. El primer párrafo de esta novela apunta hacia el poder igualmente caprichoso de la narración, pero aquí, como en Los adioses, el narrador juega con esa aparente inestabilidad y la convierte en una figura única en la obra del autor. Basándose en una arbitrariedad narrativa y una reescritura constante, estos dos textos establecen un fuerte parentesco en su exploración de las paradojas de narratividad.
\end{abstract}

\section{Palabras clave}

Onetti. Saer. Los adioses. Glosa. Narratividad. Primeros párrafos. Paratextos.

En 2005, Juan José Saer publicó una serie de textos sobre la obra de Juan Carlos Onetti, reunidos en su última colección de ensayos, Trabajos. Saer había escrito numerosos ensayos de crítica literaria durante su carrera, cubriendo una gama amplia de autores y temas, pero hasta ese momento no había dedicado ninguno al escritor uruguayo. Como para compensar por la deuda atrasada, los tres ensayos -"El soñador discreto" (lección inaugural en un coloquio Onetti en París en 2001), "Sobre Onetti y La vida breve" y "Onetti y la novela breve" (prólogo a una edición Archivos sobre las novelas cortas de Onetti, "de próxima aparición")- arrojan luz no sólo sobre la obra de Onetti, sino también sobre la del propio Saer.

\footnotetext{
* Universidad de Dalarna, Suecia.
} 
Entre otros asuntos, Saer comenta la novela corta Los adioses (1950), que compró una tarde de 1955 en una librería de Santa Fe, y destaca "el magnífico primer párrafo de la novela, que todos los aspirantes a escritores de nuestra generación sabíamos de memoria" (Saer, 2005, p. 247). "Y uno de los importantes hallazgos de ese relato", prosigue Saer, "por no decir el principal, es justamente la distancia y la posición del narrador respecto de lo que narra. La distancia y la posición, que son literalmente espaciales, trascienden ese sentido literal y traducen la fragmentariedad del conocimiento, la esencia ambigua del acontecer" (p. 247). Más de tres décadas después de la publicación de Los adioses, Saer vuelve a los temas del conocimiento parcial y la naturaleza problemática del acontecer, filtrados por la distancia y la posición, en Glosa (1986), iniciado por un primer párrafo igualmente intrigante que de alguna forma condiciona toda la lectura. La unión más fuerte entre Glosa y Los adioses, sin embargo, está en la constante reflexión sobre el acto de narrar, la autoría de una historia, el papel de la ficción, el valor de la verdad y el intercambio complejo entre autor, narrador y personaje.

En este artículo estudiaré cómo funcionan estos elementos narrativos en los respectivos textos, cuáles son sus puntos comunes y cómo Onetti y Saer reafirman lo que podría llamarse la arbitrariedad del narrador. En este caso, la arbitrariedad se refiere a dos conceptos similares, pero no idénticos: por una parte, una acción cometida por la voluntad o capricho de alguien; y por otra parte algo cometido como abuso de una autoridad. ${ }^{1}$ La combinación entre el capricho y el despotismo es algo que, como veremos, caracteriza a las dos novelas estudiadas en este artículo.

\section{Los adioses}

La novela corta Los adioses se publicó cuatro años después de la monumental La vida breve (1954), una novela que, además de narrar la génesis de Santa María, es una exploración extensa de las paradojas de la ficción y el lugar del escritor. La disposición espacial mencionada por Saer se establece en las primeras páginas de la novela. El punto de partida es un truco narrativo familiar: un desconocido entra en un bar, que en este caso también sirve de almacén y está situado en un pueblo innominado de las montañas, donde es observado por el hombre detrás de la barra. El almacenero (a quien llamaré el narrador, a pesar de que también cumple otras funciones) sólo dejará su bar unas pocas veces, y la narración toma lugar casi exclusivamente en esta área restringida. El hombre, que tiene una enfermedad pulmonar, es regularmente visitado por una mujer, y más tarde por una muchacha joven. Durante gran parte de la novela se cree que la mujer es su esposa y la joven su amante - para la gran desaprobación de la gente del pueblo- pero al final parece que la joven, en realidad, es su hija.

\footnotetext{
${ }^{1}$ La definición es del Diccionario del uso del español, de María Moliner. Oxford English Dictionary va más lejos, y describe arbitrary como algo "derived from mere opinion or preference; not based on the nature of things; hence, capricious, uncertain, varying" $y$, en segundo lugar, "unrestrained in the exercise of will; of uncontrolled power or authority, absolute; hence, despotic, tyrannical".
} 
También es importante recordar que estos lugares forman parte de la estructura espacial que posibilita la red narrativa de la novela. El narrador narra desde un cruce de información, frecuentado por observadores, personajes secundarios, y por el protagonista de la historia que cuenta el almacenero. La primera visita es lo que desencadena la instancia narrativa en primer lugar, pero como gran parte de la acción toma lugar fuera del bar, el narrador tiene que depender de informes orales para narrar una historia coherente. A su vez, el narrador es un ex-paciente que ha vivido quince años "con tres cuartos de pulmón"; éste también está marcado por una enfermedad que no sólo afecta su perspectiva del hombre, sino su narración en general. Tanto el pasado del hombre enfermo como el suyo están amputados, como si sus vidas pertenecieran exclusivamente al pueblo, resaltando las relaciones espaciales más que las temporales.

¿Qué es lo que narra, entonces, ese legendario primer párrafo, inscrito en la memoria de Saer mucho antes de la publicación de su primer libro? El texto se inicia con un subjuntivo llamativo, repetido anafóricamente en los primeros dos párrafos ${ }^{2}$ :

\begin{abstract}
Quisiera no haber visto del hombre, la primera vez que entró en el almacén, nada más que las manos; lentas, intimidadas y torpes, moviéndose sin fe, largas y todavía sin tostar, disculpándose por su actuación desinteresada. Hizo algunas preguntas y tomó una botella de cerveza, de pie en el extremo más sombrío del mostrador, vuelta la cara -sobre un fondo de alpargatas, el almanaque, embutidos blanqueados por los años- hacia afuera, hacia el sol del atardecer y la altura violeta de la sierra, mientras esperaba el ómnibus que lo llevaría a los portones del hotel viejo.

Quisiera no haberle visto más que las manos, me hubiera bastado verlas cuando le di el cambio de los cien pesos y los dedos apretaron los billetes, trataron de acomodarlos $y$, en seguida, resolviéndose, hicieron una pelota achatada y la escondieron con pudor en un bolsillo del saco; me hubieran bastado aquellos movimientos sobre la madera llena de tajos rellenados con grasa y mugre para saber que no iba a curarse, que no conocía nada de donde sacar voluntad para curarse. (Onetti, 2005, p. 723)
\end{abstract}

Estos dos párrafos son, comprensiblemente, admirados por muchos lectores y a menudo comentados en la escritura crítica sobre Los adioses como una clave a los enigmas del texto que sigue y un ejemplo excelente del estilo de Onetti. Emir Rodríguez Monegal subraya la forma en que el par de manos ocupa el lugar central de la narración, como si el narrador imitara una primera plana cinematográfica (1969, p. 181). Hugo Verani, quien ha trabajado extensamente con la obra de Onetti, observa cierta simpatía por el hombre enfermo en la mente del narrador, visible ya en las frases principales, y añade que "la figura de este narrador básico ejerce total dominio sobre el material narrativo que configura la novela" (1974, p. 176). A su vez, José Pedro Díaz apunta que "los adjetivos y los adverbios utilizados implican un ser intelectual y moral; sin embargo, sólo están referidos a las manos" (1987, p. 95, cursiva en el original).

\footnotetext{
${ }^{2}$ Me tomo la libertad de pensar que Saer en realidad se refería a los dos primeros párrafos, ya que, como ha observado la crítica, hay una fuerte unidad marcada por la anáfora y la estructura.
} 
Es importante notar, sin embargo, que mientras estos tres críticos examinan cómo el narrador se enfoca en las manos, otorgándoles atributos que de alguna manera representa al protagonista y su destino trágico en el pueblo, ninguno de ellos, ni ningún otro crítico comentando este pasaje conocido, presta atención a la palabra quisiera, aún si algunos mencionan la anáfora de pasada. Los comentarios críticos estudian este pasaje como si el quisiera simplemente no existiera -a pesar de la repetición conspicua- y el narrador se enfocara en las manos meramente como una técnica literaria. ¿Cuál podría ser la razón de esta omisión extraña -y por qué, ya que estamos en ello, el narrador quisiera no haberle visto al hombre más que las manos? Después de todo, es excepcional que el narrador abra su texto expresando un deseo hipotético, entrando en una dimensión imaginaria llena de posibilidades. El sentido del quisiera inicial depende de los motivos que cada lector encuentra para la actividad del narrador, pero en todo caso no deja de ser espectacular (y el silencio crítico confirma esto) con qué facilidad el lector acepta participar en el juego narrativo, completamente consciente de que los dados están cargados desde la primera jugada.

Naturalmente, cada uno puede especular por qué el narrador quisiera haber visto sólo las manos - tal vez porque el narrador podría prescindir de la triste figura del caballero, o quizá el resto del cuerpo no cuadra con una historia netamente tejida alrededor de las manos- pero lo importante es que el subjuntivo ocupa ese lugar como una garantía de la empresa narrativa. En primer lugar es una prueba de la fuerza de la ficción - a pesar de la repetición y los deseos explícitos- que los lectores eligen ignorar la palabra o simplemente leerla como una forma poética y elegante de iniciar la historia, entendiéndola como una parte del estilo del autor. En segundo lugar, la palabra se erige como un rastro del impulso narrativo en el centro de esta novela, y desvela el proceso que puede haber llevado a este momento: el almacenero está detrás de la barra, aburrido con la vida a su alrededor, y al entrar el hombre, el narrador instantáneamente se compromete a inventarle una historia. El indicador de esta decisión es el casi inexplicable quisiera, que, con su poder poético, enigmático y aparentemente determinado, tiene como función primaria poner en marcha la máquina narrativa. En este sentido, y como se apreciará en seguida, la situación es justamente la contraria a la que sugiere Verani, donde el narrador "ejerce total domino sobre el material narrativo"; al contrario, se lanza al proyecto narrativo sin saber muy bien qué va a ocurrir, más interesado en su poder como narrador que en contar una historia determinada de antemano.

Su relación con el material narrativo, sin embargo, nunca deja de ser ambigua. Oscilando entre la confidencia segura y la vacilación, el narrador a menudo revela un conocimiento constante y extrañamente lúcido de su actividad centrípeta sin entrar en razonamientos teóricos. Al mismo tiempo, sin embargo, parece vivir su historia como si fuera su propio público, ignorante de lo que le espera a la vuelta de la esquina. En un estado temprano de la narración, haciendo eco de la opinión de Verani, nos asegura que tiene control total de los acontecimientos: "Sabía esto, muchas cosas más, y el final inevitable de la historia cuando le acomodé la valija en la falda e hice avanzar el coche por el camino del hotel" (p. 745). No obstante, no está nada claro si "el final inevitable" significa que el narrador ya conoce el final de la historia -o si quiere decir que, sea como sea el final de la historia, será él quien lo cuente y por lo tanto la 
controlará. Por primera vez el narrador declara la intención de que la historia será suya y que sólo indirectamente pertenecerá al hombre enfermo asignado como protagonista.

A medida que el lector va entrando en el texto de Los adioses, la autoreferencialidad del narrador se va convirtiendo en el rasgo dominante. El narrador nunca olvida que es un personaje en un drama tanto vital como escrito, asignándose como el antagonista del hombre que acaba de venir a su pueblo. De vez en cuando el hombre consigue escabullirse, desapareciendo entre la gente y evadiendo la atención del narrador, pero el hombre que manda pronto le encuentra y le castiga: "me sentía responsable del cumplimiento de su destino, obligado a la crueldad necesaria para evitar que se modificara la profecía, seguro de que me bastaba recordarlo y recordar mi espontánea maldición, para que él continuara acercándose a la catástrofe" (p. 737). El hombre puede distanciarse temporalmente de su campo de visión, pero en cuanto el narrador recuerda la "espontánea maldición" pronunciada en las primeras líneas del texto, el hombre vuelve a caer bajo su hechizo, condenado a seguir cada capricho de la imaginación del narrador. En este caso la profecía realmente se autorrealiza, porque su pronunciación no sólo constituye el principio del texto, sino la misma condición y razón de ser de la historia. En el mismo acto se autorrealiza el narrador. El duelo entre los dos hombres está trucado, ya que el almacenero tiene la oportunidad de cambiar su posición post-facto y alterar los acontecimientos para que sirvan a sus propósitos. Cada uno de ellos tiene una historia que defender, $y$, aunque el narrador tenga la ventaja, lucha para dar sentido a las historias que cuenta y a la gente a la que se refiere.

Al final, el concepto de narrador es demasiado estrecho para la ambiciosa mente del almacenero; cuanto más se estudia el papel del narrador, más parece que éste no sólo está narrando la historia del hombre, aunque sea juntando trozos de información de segunda mano y rellenando los espacios en blanco, sino hasta reclamando la autoría de la misma. Si tomamos la historia como verdadera en algún sentido, a pesar de ser un obvio montage, el narrador todavía es responsable de algunas áreas completamente ficcionalizadas. Estas áreas no son inválidas; como la ficción en general, no se pueden considerar verdaderas o falsas, y además mantienen cierto grado de verosimilitud. El narrador de Los adioses nos hace confrontar una serie de preguntas sobre la naturaleza de la narración: ¿cuál es el papel del narrador de una historia? ¿Cuándo se convierte el narrador en autor? ¿Dónde está el límite entre el narrador y lo narrado?

\section{Glosa}

Éstas son también algunas de las preguntas que Saer explora en su novela de 1986, Glosa. En esta novela, dos conocidos, Ángel Leto y el Matemático, se encuentran en la calle central de una ciudad que indudablemente toma prestados muchos rasgos de la Santa Fe natal de Saer, pero que, igual que el pueblo de Los adioses, nunca se nombra. Los dos se acompañan durante una caminata por la calle, a través de veintiuna cuadras y aproximadamente cincuenta y cinco minutos, en la que se registra 
su conversación y muchas de las reflexiones simultáneas de los personajes. Un tema central de la conversación es la fiesta del cumpleaños de Washington Noriega, un personaje conocido de la ciudad, al que ninguno de los dos personajes, por distintas razones, pudo asistir. Al Matemático le han contado muchos acontecimientos y conversaciones de la fiesta, $y$ en base a lo que los dos personajes conocen de los que asistieron a la fiesta, se van formando una idea propia de la noche. (Teniendo en cuenta que el informante principal del Matemático es conocido por sus fabulaciones y sus borracheras, entre otros agravantes, la veracidad del relato no deja de ser un tanto problemática.) Aunque no se menciona en el texto, uno de los precursores de Glosa es el Simposio de Platón, un diálogo en que dos personajes hablan de la mítica fiesta en casa de Agatón, unos quince años antes, en la que Sócrates y otros simposiantes habían discutido y alabado la naturaleza misteriosa de Eros. La principal cuestión estructural del Simposio es la transmisión oral del conocimiento, que en el diálogo platónico pasa por cinco narradores distintos. Esta transmisión inestable es reflejada en Glosa (frecuentemente en frases como "dice el Matemático que le dijo Botón que dijo Tomatis", p. 51) como una forma de hacer hincapié en el estatus artificioso de cada narración.

Tanto en Glosa como en Los adioses, la paradójica reivindicación de la autoría que al mismo tiempo se declara completamente relativa se establece desde las primeras líneas del relato -o, en el caso de Glosa, hasta antes de las primeras líneas. Ya hemos visto que Saer resalta la distancia y la posición del narrador respecto a lo narrado en Los adioses, traduciendo la fragmentariedad del conocimiento, pero el autor también subraya que el inicio "anuncia la ineluctable derrota" (Saer, 2005, p. 247). La derrota y la desgracia son indudablemente unos fenómenos familiares en la narrativa de Onetti, pero una pregunta llama la atención: ¿la derrota de quién? Una posibilidad es la derrota del hombre enfermo que acaba de entrar en el almacén, aunque ya hemos visto que tal juicio es más una sentencia, reforzada por el poder narrativo que el narrador acaba de descubrir y apropiar, que una predicción sobre el futuro. En un plano más general, sin embargo, la derrota la experimenta el mismo narrador; a pesar de sus esfuerzos deductivos, a pesar de sus manipulaciones y licencias poéticas, el hombre se escurre de entre sus dedos y deja la narración incompleta.

Antes de entrar en el texto propiamente dicho, el lector de Glosa tiene que pasar por una serie de lo que Gérard Genette llama paratextos - un umbral del texto, o "d'un 'vestibule' qui offre à tout un chacun la possibilité d'entrer, ou de rebrousser chemin. 'Zone indécise' entre le dedans et le dehors, elle-même sans limite rigoureuse, ni vers l'intérieur (le texte) ni vers l'extérieur (le discours du monde sur le texte)" (1987, p. 8). Esta periferia textual, ni perteneciente al texto ni completamente excluida, es un espacio de transición y transacción, donde las intenciones autoriales se topan con el mundo exterior. ${ }^{3}$ J. Hillis Miller aclara que "'para' is a double antithetical

3 Jacques Derrida, refiriéndose al parergon de Kant, lleva la discusión un paso más lejos y destaca que la necesidad de distinguir entre el sentido interno de una obra de arte y el contexto alrededor de la obra artística "organizes every philosophical discourse on art, the meaning of art, and meaning itself" (Derrida, 1979, p. 12). 
prefix signifying at once proximity and distance, similarity and difference, interiority and exteriority" (Miller, 1979, p. 219).

El inicio de Glosa no es, pues, del todo fácil de precisar; en realidad hay cuatro puntos de partida distintos, que todos remiten a otro lugar y hacen que la lectura se vuelva sobre sí misma. El primero está en el mismo título: en el uso general, glosa quiere decir una "explicación o comentario de un texto oscuro o difícil de entender", un comentario hecho entre líneas de un texto como una suerte de interpretación. En la terminología literaria, más específicamente, la glosa es una composición poética de varios versos, muchas veces preexistentes, que luego se comentan y se expanden en una serie de estrofas donde los versos iniciales se repiten al final de cada estrofa. El vocablo glosa tiene un sentido notoriamente polifacético en un texto que trabaja constantemente con la interpretación, reescritura y recontextualización del material intra-textual. El término también apunta, de igual forma, hacia otros textos literarios, y en ese sentido es evidente que la glosa es tanto una lectura como una escritura. Así, la novela de Saer es sobre una glosa, pero por otra parte la novela es esa misma glosa.

La palabra del título, al igual que en muchas de las narraciones de Saer, no se menciona ni una sola vez en el texto, así que en vez de referirse a algo concreto, el título actúa como una sobreestructura o una caja de resonancia.

La lectura se aprecia en el siguiente paratexto, la dedicación:

A

Michel, Patrick, Pierre Gilles,

que practican tres

ciencias verdaderas,

la gramática, la homeopatía, la administración,

el autor les dedica,

por las sobremesas de los domingos,

esta comedia:

but then time is your misfortune father said.

Uno podría pensar que las historias de exilio, desapariciones, violencia y muerte que circulan alrededor de la narración harían del texto más una tragedia que una comedia. La cita de Faulkner, extraída del capítulo correspondiente a Quentin en The Sound and the Fury, encarna esta superimposición de lo cómico y lo trágico en la misma dedicación. Sobre esas sobremesas dominicales, parecidas a la fiesta en el centro de Glosa, la sombra del tiempo se alarga: "Father said a man is the sum of his misfortunes. One day you'd think misfortune would get tired, but then time is your misfortune Father said." (Faulkner, 1994, p. 66). Saer aclara la cuestión de la comedia y la tragedia entrelazada en "Líneas del Quijote" (en La narración-objeto), donde vuelve a citar la misma cita faulkneriana y habla de la moral del fracaso en la literatura occidental: "A pocas excepciones que existen en la literatura moderna, podríamos definirlas como comedias, pero hay que ser conscientes de que en las verdaderas comedias [...] la sombra amarga de fracaso se proyecta continuamente sobre la intriga" (Saer, 1999, p. 47). Tal y como Saer destaca sobre los primeros párrafos de 
Los adioses, el fracaso y la derrota se imponen sobre la narración que está a punto de comenzar.

Un tercer paratexto, de una naturaleza más compleja y un significado polifacético, es el poema que constituye el epígrafe:

En uno que se moría

mi propia muerte no vi, pero en fiebre y geometría se me fue pasando el día y ahora me velan a mí.

El poema pasa por varios estadios según progresa la narración. Tal y como aparece en el epígrafe no hay ninguna indicación de que el poema no tiene que ser atribuido al autor real del libro, y no es hasta la mitad de la segunda parte de la novela que sabemos que el poema tiene un autor ficticio, Carlos Tomatis. Leto y el Matemático acaban de toparse con un Tomatis muy malhumorado que saca un papel y lee el poema a sus dos interlocutores. Después de leerles el poema por segunda vez a Leto y al lector real, Tomatis regala el papel del poema al Matemático, que a su vez lo mete en su cartera. Con el paso del tiempo, el poema pasa de lenguaje hablado a texto escrito, y de texto escrito a símbolo; cuando el Matemático está en el avión camino a su exilio sueco en 1979 , saca la hoja y recuerda que nunca ha sido capaz de tirarla. El papel se ha transformado en un vestigio - no de Tomatis o su poesía, sino de la mañana soleada en que las palabras sonaron en el aire.

Pero para ser exactos, ¿quién convierte el poema en epígrafe, y por qué? ¿Cómo trasciende su estatus puramente ficticio para ser uno de los umbrales de la ficción? En el caso de Glosa, el epígrafe reivindica el poema de Tomatis de la existencia triste como artefacto material, convirtiéndola a ser el portal sobre el que cada lector, como entrando al templo de Delfos, tiene que reflexionar. En ese sentido, el poema se parece a la estrofa al principio de la glosa poética, donde el texto entero de la novela viene a ser la expansión y la clarificación de las primeras cinco líneas. ${ }^{4} \mathrm{Si}$ se desautoriza de esa forma, ¿quién sería el yo del poema? Según la novela misma, el poema parece describir la situación de Leto más que la de Tomatis; durante la caminata por la ciudad, Leto está obsesionado por el suicido de su padre, ese "suicida insolente", inconsciente de que repetirá la muerte de su padre en el futuro.

Por su nombre, Leto viene a ser el suplente de lector, como sugieren algunos críticos (Fonsalido, 2005, p. 37), lo cual le convertiría en el intérprete principal como un suplemento del lector real. Más probable, entonces, es que el yo del poema corresponde tanto a Leto como al lector implícito de Glosa, colocando este último en una posición desde la cual la novela puede ser leída y borrando la frontera -haciendo eco de la admonición de "conócete a ti mismo"- entre el sujeto y el objeto. El enlace entre la muerte del otro y la propia es simbolizado por otra pareja de antagonistas,

4 El mismo Saer sugiere esa posibilidad: "Y la novela, de algún modo, con cinco versos que aparecen como epígrafe, opera un sistema de reincorporación del sentido de ese poema diseminado en el texto" (Piglia y Saer, 1995, p. 13). 
"fiebre y geometría", pero, en realidad, es el primer ejemplo de una extensa estructura oposicional alrededor de la cual se construye la novela. ${ }^{5}$ Consecuentemente, ¿puede el poema del epígrafe caracterizarse como una copia del poema mecanografiado de Tomatis en el texto, y puede llamarse original si está escrita por un personaje ficticio? ¿Cuál es su estatus original, si va de poesía a artefacto y vuelve a ser poesía? Si es una mise-en-abîme, algo que encontramos varias veces en Glosa, ¿cómo entender una situación que tanto precede como está incluida en el texto? El hecho de que no hay una respuesta clara a estas preguntas apunta hacia la figura del bucle en el centro de esta narración, donde no es posible distinguir entre original y copia.

\section{El primer párrafo como relectura}

Con estos paratextos, que no dejan de socavar la autoridad del narrador, llegamos a lo que parece ser el verdadero inicio, un párrafo que condiciona la lectura de igual forma que los primeros párrafos de Los adioses:

Es, si se quiere, octubre, octubre o noviembre, del sesenta o del sesenta

y uno, octubre tal vez, el catorce o el dieciséis, o el veintidós o

el veintitrés tal vez, el veintitrés de octubre de mil novecientos sesenta

y uno pongamos -qué más da. (Saer, 1988, p. 13)

Es con este párrafo inicial que Glosa se mide con Los adioses; como una relectura, comentario o, si se quiere, una glosa. No tanto porque las dos novelas sean comparables punto por punto -ya que hay demasiadas diferencias- sino porque comparten una forma de ver la fragmentariedad de lo conocido, la verdad a través de la ficción, y el poder caprichoso del narrador y su modo de acercarse a lo narrado. La derrota que Saer ve en las primeras líneas de la novela de Onetti ya se ha anunciado en varios de los paratextos, pero también aquí se relaciona tanto con el fracaso humano como con la imposibilidad de conocer la verdad sobre el pasado. Como en el caso del almacenero onettiano, quien ya desde el principio está fascinado por el poder de sus palabras, en estas primeras líneas se duda en qué día tiene lugar la narración y al mismo tiempo se fija una fecha exacta. La delimitación es arbitraria, aunque, no obstante, una delimitación de la que dependen todas las otras fechas de la novela.

Detrás de esta indecisión y las perspectivas fragmentarias que se presentan hay una figura única en la obra de Saer: el narrador extraño e irónico en el centro de la narración. El hecho de que este narrador mezclará omnisciencia y arbitrariedad está

\footnotetext{
${ }^{5}$ A primera vista, no es difícil ver a los dos protagonistas como personificaciones. La geometría sería representada por el Matemático; rico, físicamente fuerte, vestido todo de blanço, y apodado así por lo que Tomatis considera su confianza exagerada en las ciencias exactas. Ángel Leto, por otra parte, incorpora otro lado de la existencia: es pobre, bajo, vestido con unos vaqueros baratos, y tiene una relación más bien emocional con el arte y la política. Raquel Linenberg-Fressard, la primera investigadora en analizar la pareja de fiebre y geometría, ve la fiebre como una fuerza centrífuga, en último lugar llevando al exilio, la diáspora y la muerte, mientras la geometría es su contraparte centrípeta y organizadora (1991, p. 105-106).
} 
claro desde las primeras líneas, donde se establece el contra o especial entre narrador y lector. En las partes diegéticas del texto que sigue, constantemente interrumpidas por marcas conversacionales vacilantes - "ino?" "decíamos," "si se quiere," "a decir verdad -es un decir, ¿no?"- el narrador es capaz de penetrar libremente en la mente de los personajes e interpretar signos y expresiones. A pesar de ello se muestra inseguro de su papel como narrador, constantemente declarando su consciencia de que un narrador omnisciente es una convención literaria y lingüística como cualquier otra. Nicolás Lucero estudia el papel del narrador con gran detalle, y define el estatus extraño de esta omnisciencia:

La omnisciencia del narrador de Glosa es, sin embargo, singular. En muchos sentidos se trata de una omnisciencia hiperbólica, exagerada, que contraviene, aunque suene paradójico, los límites verosímiles de los conocimientos que puede tener un narrador sobre lo que cuenta. En lugar de garantizar una visión abarcadora de los acontecimientos y los sentimientos, la rara omnisciencia del narrador está puesta al servicio de la fragmentación del texto (2006, p. 203).

La función de la omnisciencia de este narrador es, entonces, contraria al texto realista clásico; sin duda sabe más que sus personajes, aunque al mismo tiempo parece tener una visión fragmentada tanto de sus vidas interiores como del cumpleaños de Washington Noriega. Este "servidor", como a veces se autoproclama para mantenerse en el fondo (aunque el resultado es, a decir verdad, el contrario), no duda en presentar su propia opinión sobre el comportamiento de los personajes.

Lo que es todavía más llamativo es cómo el narrador está más que seguro sobre el futuro de los personajes, y cómo ocasionalmente interrumpe el presente narrativo - que a menudo mira hacia el pasado- con prolepsis largas y a menudo vertiginosas. El futuro vislumbrado es el subtexto trágico del presente narrativo cómico. De los tres amigos que caminan por San Martín una mañana soleada de 1961, sabemos que Leto se hará guerrillero y se verá obligado, finalmente, a tragar la pastilla suicida que ha llevado encima durante sus años de lucha armada. El Matemático se irá al exilio a finales de la época oscura y violenta de los 1970, después del asesinato de su esposa. Tomatis, por su parte, caerá en una depresión severa, profundamente afectado por las desapariciones, torturas y muertes a su alrededor. Cada uno está como al borde de un abismo metafísico del cual saben muy poco durante la mañana de la caminata. Las corrientes de la Historia fluyen hacia la muerte y la nada, pero se suspenden debido al arte y la magia del eterno presente. A pesar de la omnipresencia fatal de la tragedia, a pesar de un pasado inalcanzable y un futuro de aniquilación, la literatura sigue siendo posible entre la primera página y la última, entre la primera cuadra y la vigésima primera: "En ese marco estricto, la novela se deja escribir; el instante de delicia (que es también el tiempo de la lectura), esa verdadera 'epifanía' se produce, efímero pero eficaz" (Premat, 2002, p. 251). Con esto volvemos al poema del paratexto; aunque uno no pueda o no quiera reconocer su propia muerte en la cara del otro, el tiempo, ineluctablemente, la traerá. La voz del narrador se esconde tras la máscara del poeta: "El saber del narrador es comparable al de este poema de Tomatis, quien, entre todos los personajes, ya sabe y sabe que sabe" (Sarlo, p. 5). Aún conociendo el subtexto trágico, el narrador narra los pensamientos y las acciones de aquella mañana soleada 
de primavera en todo su detalle minucioso, consciente de que la historia de los personajes ya está escrita.

En los dos textos estudiados en este artículo, ambos autores escriben a partir de una serie de experiencias de la literatura: la conciencia de la derrota vital y literaria, la conciencia de la posición espacial para el desarrollo de la historia y la paradoja de un narrador que cuenta una historia al mismo tiempo escrita y en el proceso de escribirse. La arbitrariedad de los narradores, con un poder narrativo que vacila y al mismo tiempo afirma su autoridad despótica, les convierte en los verdaderos protagonistas.

\section{Bibliografía}

DERRIDA, Jacques. "The Parergon." October 9. Summer (1979, p. 3-41).

DÍAZ, José Pedro. "Sobre Juan Carlos Onetti." Juan Carlos Onetti. Ed. Hugo J. Verani. Madrid: Taurus, 1987.

FAULKNER, William. The Sound and the Fury. Ed. David Minter. New York \& London: W. W. Norton and Company, 1994.

FONSALIDO, María Elena. "Una lectura de Glosa, de Juan José Saer." Del recuerdo de la voz: homenaje a Juan José Saer. Los polvorines: Universidad nacional del general Sarmiento, 2005.

GENETTE, Gérard. Seuils. París: Éditions du Seuil, 1987.

LINENBERG-FRESSARD, Raquel. "'Fiebre' y 'geometría' en Glosa de Juan José SAER." Le roman hispano-américain des années 80. Les cahiers du CRIAR. Ed. Claude Cymerman. Vol. 11. Rouen: Publications de I'Université de Rouen, 1991. 103-108. LUCERO, Nicolás. "Zona y exterioridad: personaje, narrador y diálogo en la obra de Juan José Saer." Tesis doctoral. University of Iowa, 2006.

MILLER, J. Hillis. "The Critic as Host." Deconstruction and Criticism. Ed. Harold Bloom et al. New York: The Seabury Press, 1979.

ONETTI, Juan Carlos. Juan Carlos Onetti: Obras completas. Novelas (1939-1954). Introducción de Dolly Onetti. Prólogo de Juan Villoro. Ed. Hortensia Campanella. Vol. I. Barcelona: Círculo de lectores/Galaxia Gutenberg, 2005.

PIGLIA, Ricardo; SAER, Juan José. Diálogo. Ed. Sergio Delgado. Santa Fe, Argentina: Universidad Nacional del Litoral, 1995.

PREMAT, Julio. La dicha de Saturno: escritura y melancolía en la obra de Juan José Saer. Rosario: Beatriz Viterbo Editora, 2002.

RODRÍGUEZ Monegal, Emir. Narradores de esta América. Ensayos. Montevideo: Alfa, 1969. 
SAER, Juan José. Glosa. 1986. 1a ed. Barcelona: Ediciones Destino, 1988.

. La narración-objeto. 1999. Los tres mundos. Ensayo. Buenos Aires: Seix

Barral, 1999.

. Trabajos. Buenos Aires: Seix Barral, 2005.

SARLO, Beatriz. "La política, la devastación." Sin publicar.

VERANI, Hugo J. "En torno a Los adioses de Juan Carlos Onetti." Homenaje a Juan

Carlos Onetti: variaciones interpretativas en torno a su obra. Ed. Helmy F. Giacoman.

Salamanca: Anaya, 1974. 161-180.

\section{Abstract}

The point of departure for this article is an essay, published in 2005, in which Juan José Saer comments on the well-known opening paragraphs of Juan Carlos Onetti's Los adioses (1954). In these first paragraphs of the novel, the reading contract is established between narrator and reader, but its foundations are shaky: at the same time as the narrator claims authorship (and authority) in the most despotic terms, he also declares it completely relative. In this way, a number of important questions are posed, of which one is crucial: what is the role of the narrator of a story? The same question characterizes Juan José Saer's Glosa (1986), a novel which centers on the (unreliable) transmission of narrative. The narrator's unstable authority is undermined by a series of important paratexts, which all resound throughout the novel. The opening paragraph of the novel points to a similarly capricious power of narrative, but here, as in Los adioses, the narrator plays with the apparent instability and becomes a unique figure in the author's oeuvre. Grounded in a narrative arbitrariness and a constant rewriting, these two texts establish a strong bond in their exploration of the paradoxes of narrativity.

\section{Keywords}

Onetti. Saer. Los adioses. Glosa. Narrativity. First paragraphs. Paratexts. 